\title{
Hyperbaric Oxygen for Osteomyelitis in a Compromised Host
}

\author{
Lawrence A. Delasotta ${ }^{*}$, , Andrew Hanflik ${ }^{1}$, Griffin Bicking ${ }^{2}$ and William J. Mannella ${ }^{3}$ \\ ${ }^{1}$ Kingsbrook Jewish Medical Center, Department of Orthopedic Surgery, Brooklyn, NY 11203, USA \\ ${ }^{2}$ Crozer-Keystone Health System, Vascular Surgery and Endovascular Intervention Fellow, Upland, PA 19013, USA \\ ${ }^{3}$ Department of Surgery, Crozer-Keystone Medical Center, Upland, PA 19013, USA
}

\begin{abstract}
Post-traumatic osteomyelitis is a rare but debilitating complication of closed fractures. Most infections can be resolved with aggressive debridement, intravenous antibiotics, and hardware removal. However, the eradication of infection can be challenging in a patient with multiple comorbidities. Refractory infection may require suppressive therapy or amputation to control the disease. Improvements in care have led to improved survival for this population, posing new challenges in their post-traumatic care. We report on the successful use of hyperbaric oxygen therapy as an adjunct in a compromised host with recurrent post-traumatic osteomyelitis despite aggressive debridement, removal of instrumentation, and several courses of intravenous antibiotic therapy. Hyperbaric oxygen may be considered as an adjunct to standard treatment protocols for refractory osteomyelitis in compromised hosts.
\end{abstract}

Keywords: Hyperbaric oxygen, infection, trauma, osteomyelitis, vascular disease.

\section{INTRODUCTION}

Acute post-traumatic osteomyelitis is uncommon following percutaneous treatment of a closed long bone fracture. Even less common is a late presenting infection. Superficial (cortex) and deep (cortex and marrow) infection have been estimated at 3\% and $1 \%$, respectively following reamed nailing of the lower extremity [1].

Standard treatment for osteomyelitis includes extensive irrigation and debridement, intravenous antibiotics, and reconstruction where necessary. Clearance of infection can be a challenge and is often impeded by systemic comorbidities like diabetes mellitus, obesity, and/or peripheral vascular disease, all of which are believed to affect perfusion of blood and antibiotics through soft tissue and bone [2]. Refractory infection may require suppressive therapy or amputation to control the disease. Blunt trauma in this patient population has historically led to a poor prognosis; however, recent advances in modern trauma and acute surgical treatments have led to increased survivorship, posing new post-traumatic treatment challenges [3-7].

Hyperbaric oxygen (HBO) is a treatment modality that enhances blood perfusion to and improves innate immunity at the site of injury. It encourages osteogenesis, neovascularization, and collagen production [8-11]. Despite studies to suggest the resolution of chronic osteomyelitis in diabetic patients [12], the evidence base for the trauma patient is disputable [13-15]. The purpose of this case study is to suggest that a vasculopathic patient poorly fit for surgical intervention with refractory osteomyelitis may benefit from early $\mathrm{HBO}$ as an adjunct to standard treatment.

*Address correspondence to this author at the Kingsbrook Jewish Medical Center, 585 Schenectady Avenue, Brooklyn, NY 11203, USA;

Tel: (718) 604 - 5483; Fax: (718) $604-5575$;

E-mail: LawrenceDelasotta@Gmail.com

\section{CASE REPORT}

A sixty-four year old male presented to the emergency room after being struck by a motor vehicle. He was noted to have significant dyspnea with decreased breath sounds, midline vertebral tenderness, and a shortened, pulseless left lower extremity. The patient immediately received a chest tube for a pneumothorax, and was subsequently intubated. A popliteal artery and vein transection with ipsilateral subtrochanteric fracture (Fig. 1) were also noted, and an emergent direct anastomosis and three compartment fasciotomy were performed. Further workup revealed compression fractures at L1 and L3 and transverse process fractures at L2/3, all minimally displaced, not requiring operative intervention.

The patient's medical history was significant for peripheral vascular disease, prior myocardial infarction (with subsequent bypass graft $\mathrm{x} 4$ ) and sick sinus syndrome (with pacemaker placement). One week after his initial injury, the patient was medically stable, and underwent an uncomplicated cephalomedullary nailing of his femur fracture (Fig. 2).

On postoperative day five the patient suffered from both pneumonia and fasciotomy site infection. He returned to the operating room for multiple washouts, with deep cultures demonstrating methicillin-resistant Staphylococcus aureus (MRSA) treated with IV vancomycin. The patient's femoral incisions healed uneventfully, and he was discharged home on hospital day 25 .

Approximately three months after discharge, the patient presented to his primary care provider with a new left leg discomfort and a warm mass on the anterior aspect of his thigh. The pain worsened, and the mass enlarged, becoming erythematous and indurated. Oral Amoxicillin-Clavulanate was started and the patient was sent for a CT scan, which revealed a fluid collection between the sartorius and vastus 
medialis muscles, in close proximity to the now-healed fracture site. The lesion extended to the distal medial thigh (Fig. 3). The infection source was unknown, but all operative interventions used sterile technique. These authors believe the source was likely of earlier hematogenous origin from the pneumonia and/or fasciotomy site infections.

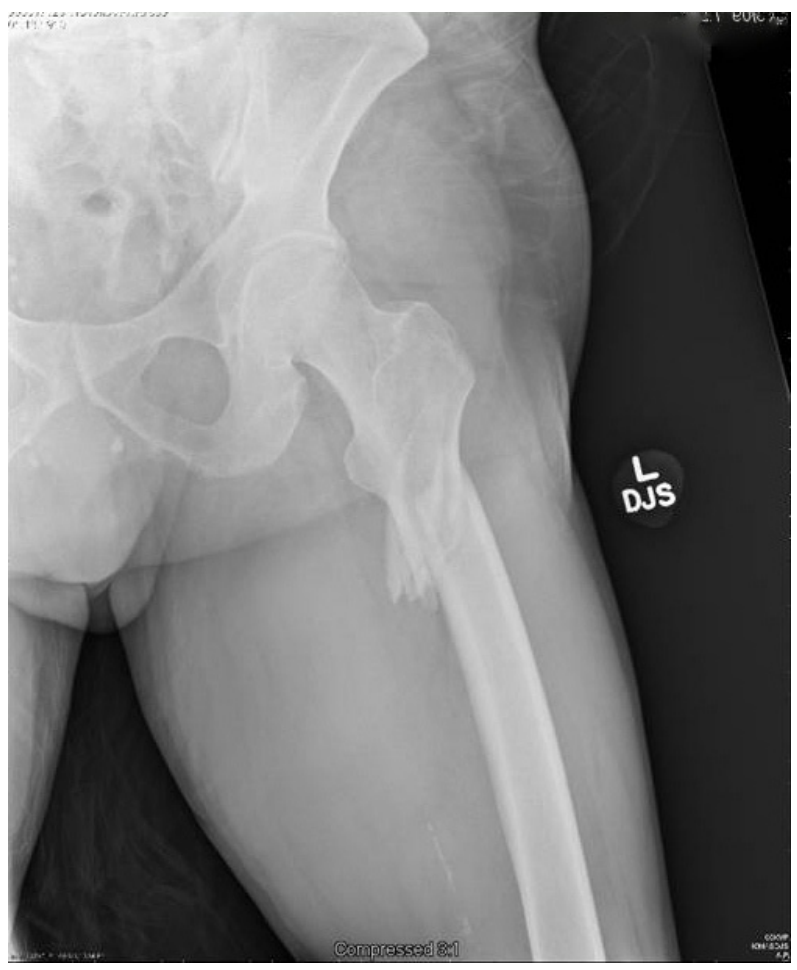

Fig. (1). A/P of proximal left lower extremity. Note the subtrochanteric spiral fracture.

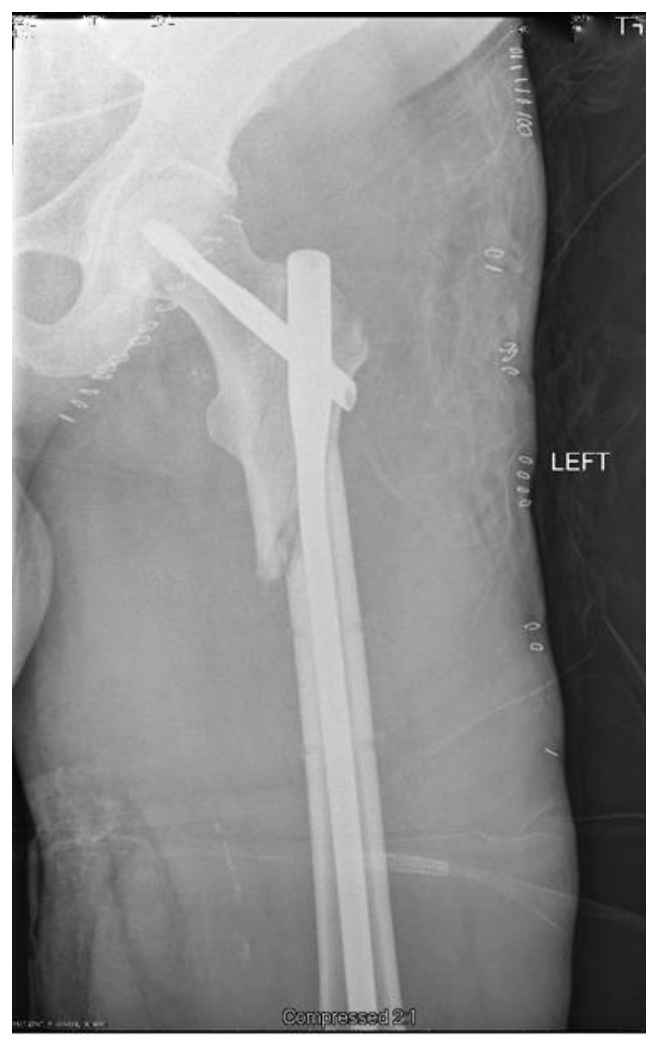

Fig. (2). A/P of femur. Note the left femur Cephalomedullary nail.

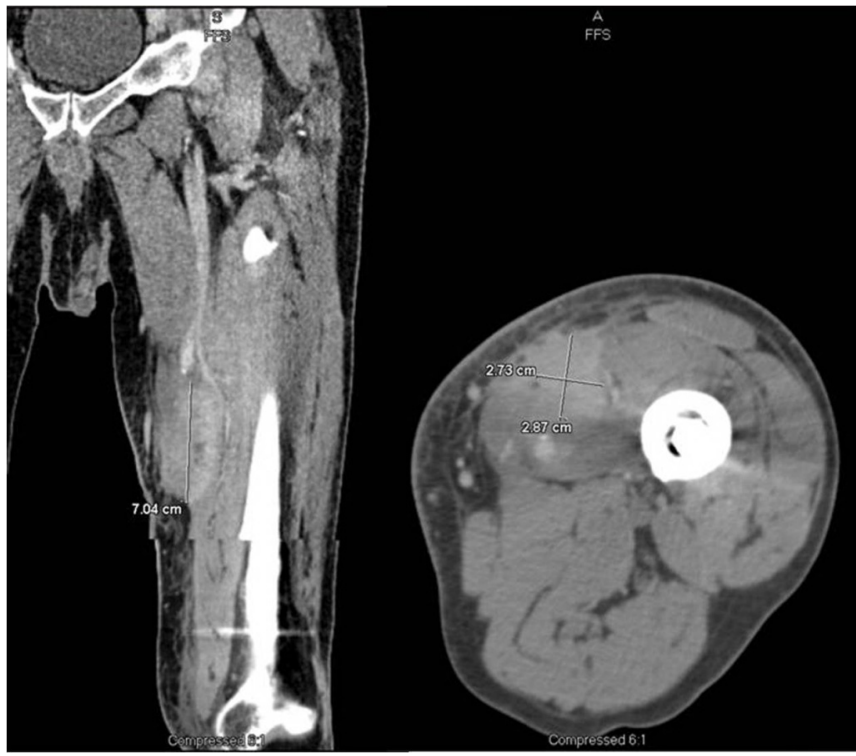

Fig. (3). CT of left lower extremity with IV contrast. Note poorly defined heterogeneous high attenuation substance in the soft tissues of the medial mid left thigh.

The patient was taken to the operating room where the abscess was drained. A sinus tract extended to the previous fracture site. Gaps of necrosis and soft tissue were isolated to approximately $4 \times 5 \mathrm{~cm}$ of bone. It was decided to remove the cephalomedullary nail. The necrotic bone and soft tissue were debrided back to healthy margins and appeared stable enough intraoperatively not to necessitate further fixation. The wounds were packed with betadine-soaked gauze sponges and allowed to heal by secondary intent while the patient received six weeks of IV vancomycin for cultures that grew MRSA.

After a full course of antibiotics the patient experienced progressively worsening recurrent thigh pain and swelling. A bone scan demonstrated bony uptake, and a CT scan demonstrated a collection in the vastus lateralis (Fig. 4). The patient was again brought to the operating room for aggressive debridement. Intraoperative cultures grew MRSA. After continued symptoms despite two additional debridements and intravenous antibiotics, the decision was made to utilize adjunctive hyperbaric oxygen therapy. Following partial closure of his wound he began a regimen of thirty HBO sessions at two atmospheres (atm) of pressure. After beginning therapy, a rapid improvement was noted in his condition, and his wound closed soon thereafter. The patient has remained asymptomatic for ten months following the final $\mathrm{HBO}$ treatment.

\section{DISCUSSION}

This case reports the resolution of refractory osteomyelitis (Cierny-Mader classification: IIC) using hyperbaric oxygen (HBO) as an adjunct treatment in a post multi-system blunt trauma patient with numerous severe comorbidities. This is an expanding patient population due to modern acute surgical care improvements [3].

Chen et al. noted that HBO in addition to both an adequate surgical debridement and appropriate antibiotic selection for chronic osteomyelitis of the femur was efficacious. Their case series consisted of 13 patients who 
underwent a mean 4.6 surgeries, were 40yoa (range: 21-61), and had Cierny-Mader classifications of III-IVA or B. The presented case suggests that a patient with significant medical comorbidities (Cierny-Mader classification, IIC), and thus a poor surgical candidate, may benefit from early initiation of HBO therapy. This case utilized earlier HBO treatment as a means to delay surgical intervention, and as a corollary likely contributed to disease resolution in a compromised host.

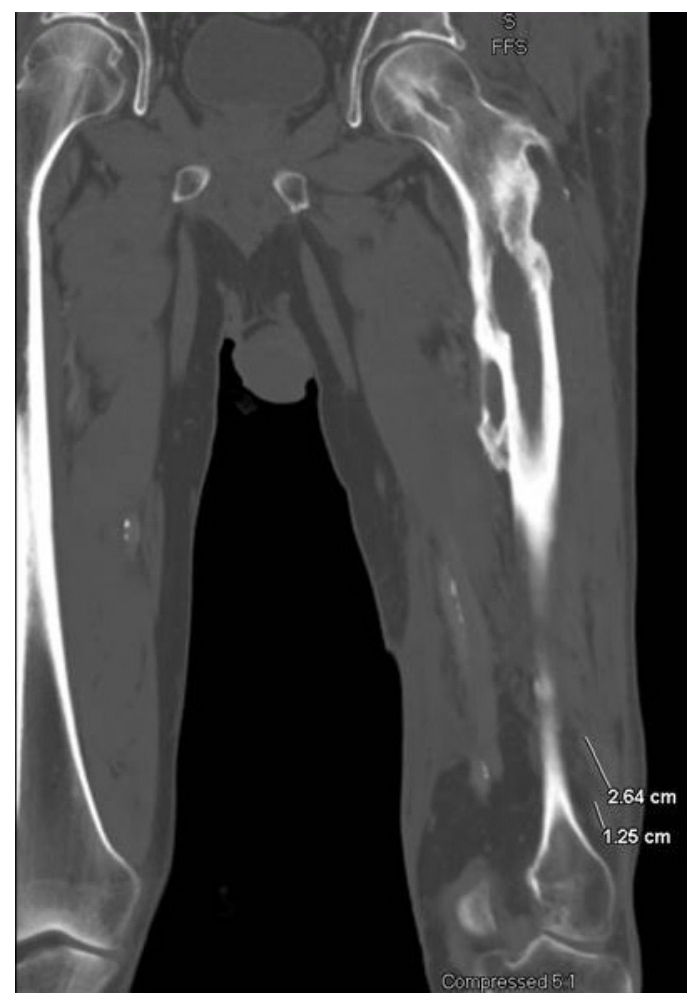

Fig. (4). Coronal CT of left lower extremity. Note former heterogeneous high attenuation mass-like areas in medial soft tissue of thigh no longer present. There are two new ill-defined areas of peripheral rim enhancement and central hypodensity in the distal vastus lateralis, suspicious for abscesses.

Ahmed et al. reviewed five cases of spinal osteomyelitis that underwent HBO therapy who had numerous risk factors for poor healing. Their HBO treatment resulted in the resolution of infection in five of six patients. They concluded that HBO therapy may be an efficacious modality as an adjunctive treatment to standard medical and surgical therapy in the patient with multiple comorbidities in relapsing primary spinal osteomyelitis [16].

$\mathrm{Yu}$ et al. evaluated the use of $\mathrm{HBO}$ as an adjunctive treatment for sternal infection and osteomyelitis after sternotomy and cardiothoracic surgery. This retrospective analysis compared six patients who had HBO to six who didn't. They reported no treatment-related complications. The intensive care unit length of stay in patients who received $\mathrm{HBO}$ was approximately forty-days shorter. In addition, duration of invasive and non-invasive positive pressure ventilation was also decreased. Furthermore, they also noted that hospital mortality was lower in HBO patients [17].

The plausibility of and few side effects from HBO therapy make it sensible as an adjunct treatment in osteomyelitis $[18,19]$. Thus the purpose of this case study is to suggest that a vasculopathic patient poorly fit for surgical intervention with refractory osteomyelitis may benefit from early $\mathrm{HBO}$ as an adjunct to standard treatment $[16,17,20]$.

HBO therapy may place an increasingly important role in the Cierny-Mader classification of II-IVC patients who are high risk surgical candidates. We suggest a combination of instrumentation removal, aggressive surgical irrigation and bone debridement, intravenous antibiotic treatment, and early hyperbaric oxygen therapy for a compromised host who develops recurrent osteomyelitis. Future study of stage II-IVC patients are warranted as this is likely an expanding patient population with a paucity of medical evidence.

\section{CONFLICT OF INTEREST}

The authors confirm that this article content has no conflict of interest.

\section{ACKNOWLEDGEMENTS}

\author{
Declared none.
}

\section{REFERENCES}

[1] Coles CP, Gross M. Closed tibial shaft fractures: management and treatment complications. A review of the prospective literature. Can J Surg 2000; 43(4): 256-62.

[2] Gottrup F. Oxygen in wound healing and infection. World J Surg 2004; 28(3): 312-5.

[3] Cuschieri J, Johnson JL, Sperry J, et al. Benchmarking Outcomes in the Critically Injured Trauma Patient and the Effect of Implementing Standard Operating Procedures. Ann Surg 2012; 255(5): 993-9.

[4] Dinh MM, Roncal S, Byrne CM, Petchell J. Growing trend in older patients with severe injuries: mortality and mechanisms of injury between 1991 and 2010 at an inner city major trauma centre. ANZ J Surg 2013; 83(1-2): 65-9

[5] Parker MJ, Handoll H. Gamma and other cephalocondylic intramedullary nails versus extramedullary implants for extracapsular hip fractures in adults. Cochrane Database Syst Rev 2005; 4: 93.

[6] Bradburn E, Rogers FB, Krasne M, et al. High-risk geriatric protocol: Improving mortality in the elderly. J Trauma Acute Care Surg 2012; 73(2): 435-40.

[7] Søreide K, Krüger AJ, Vårdal AL, Ellingsen CL, Søreide E, Lossius HM. Epidemiology and contemporary patterns of trauma deaths: changing place, similar pace, older face. World J Surg 2007; 31(11): 2092-103.

[8] Mendel V, Reichert B, Simanowski H, Scholz H. Therapy with hyperbaric oxygen and cefazolin for experimental osteomyelitis due to Staphylococcus aureus in rats. Undersea Hyperb Med 1999; 26(3): 169-74.

[9] Park M, Muhvich K, Myers R, Marzella L. Hyperoxia prolongs the aminoglycoside-induced postantibiotic effect in Pseudomonas aeruginosa. Antimicrob Agents Chemother 1991; 35(4): 691-5.

[10] Hehenberger K, Brismar K, Lind F, Kratz G. Dose dependent hyperbaric oxygen stimulation of human fibroblast proliferation. Wound Repair Regen 1997; 5(2): 147-50.

[11] Marx RE, Ehler WJ, Tayapongsak P, Pierce LW. Relationship of oxygen dose to angiogenesis induction in irradiated tissue. Am J Surg 1990; 160(5): 519-24.

[12] Kranke P, Bennett M, Roeckl-Wiedmann I, Debus S. Hyperbaric oxygen therapy for chronic wounds. Cochrane Database Syst Rev 2004; (2): CD004123.

[13] Villanueva E, Bennett M, Wasiak J, Lehm J. Hyperbaric oxygen therapy for thermal burns. Cochrane Database Syst Rev 2004; (3): CD004727.

[14] Bennett M, Stanford R, Turner R. Hyperbaric oxygen therapy for promoting fracture healing and treating fracture non-union. Cochrane Database Syst Rev 2005; (1): CD004712.

[15] Esterhai Jr J, Pisarello J, Brighton CT, Heppenstall RB, Gellman H, Goldstein G. Adjunctive hyperbaric oxygen therapy in the 
treatment of chronic refractory osteomyelitis. J Trauma 1987; 27(7): 763-8.

[16] Ahmed R, Severson III MA, Traynelis VC. Role of hyperbaric oxygen therapy in the treatment of bacterial spinal osteomyelitis. J Neurosurg Spine 2009; 10(1): 16-20.

[17] Yu WK, Chen YW, Shie HG, Lien TC, Kao HK, Wang JH. Hyperbaric Oxygen Therapy as an Adjunctive Treatment for Sternal Infection and Osteomyelitis after Sternotomy and Cardiothoracic Surgery. J Cardiothorac Surg 2011; 6(1): 141-6.
[18] Leach R, Rees P, Wilmshurst P. Hyperbaric oxygen therapy. BMJ 1998; 317(7166): 1140-3.

[19] Weaver LK, Churchill S. Pulmonary Edema associated with hyperbaric oxygen therapy. Chest 2001; 120(4): 1407-9.

[20] Chen CE, Ko JY, Fu TH, Wang CJ. Results of chronic osteomyelitis of the femur treated with hyperbaric oxygen: a preliminary report. Chang Gung Med J 2004; 27(2): 91-7.

Received: October 23, 2012

Revised: March 27, 2013

Accepted: March 28, 2013

(C) Delasotta et al.; Licensee Bentham Open.

This is an open access article licensed under the terms of the Creative Commons Attribution Non-Commercial License (http://creativecommons.org/licenses/by-nc/3.0/) which permits unrestricted, non-commercial use, distribution and reproduction in any medium, provided the work is properly cited. 